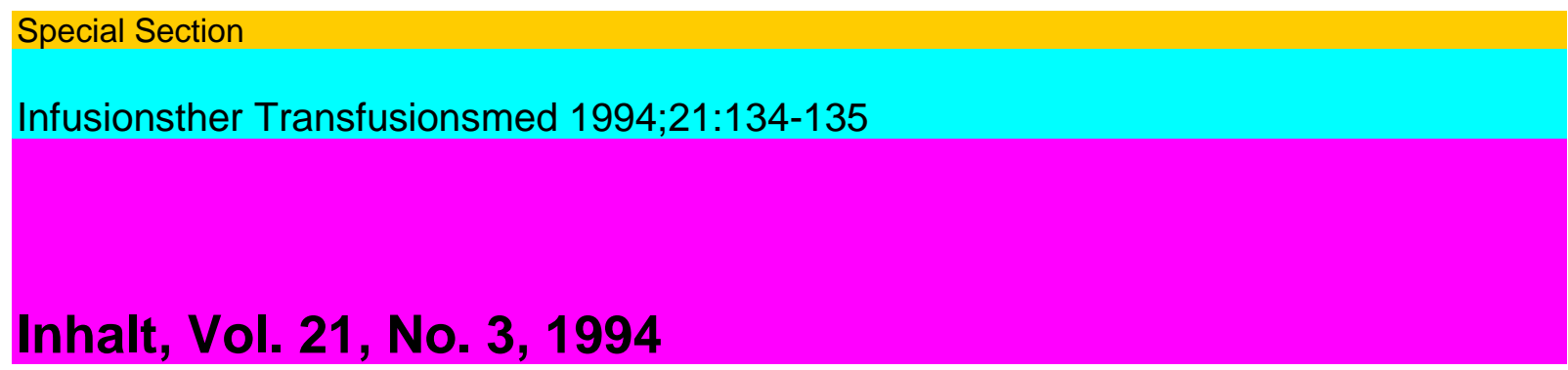

\title{
Editorial Editorial
}

Das Blutvolumen: Kein einheitliches Volumen, sondern dieBlood Volume: Not one Single

Volume but the Sum of

Summe zweier Volumina mit unterschiedlichem Mischungs- $\quad$ Two Volumes with Various

Mixing in Different Parts of the

verhalten in verschiedenen Teilen des Kreislaufs Circulation

Haller, M., Finsterer, U. 136 Haller, M., Finsterer, U. 136

Originalarbeiten Original Paper

Blutvolumenbestimmung mit Fluorescein-Natrium und Blood Volume Determination with

Sodium Fluorescein and

radioaktivem Chrom - Ein klinischer Methodenvergleich Radioactive Chromium - A Clinical Comparison of Methods

Lauermann, I., Wilhelm, G., Kirchner, E 138 Lauermann, I., Wilhelm, G., Kirchner, E 138

Auswertung des Anti-HCV-ELISA-Screening-Tests bei Evaluation of Anti-HCV ELISA

Seropositivity in

freiwilligen Blutspendern: Ein Vorschlag für eine neue Voluntary Blood Donors: A Proposal for Donor Counseling

Spenderberatungsstrategie Strategies

Beckers, E. A. M., Thijssen, E M.H. I, Nijdam, T., Beckers, E. A.M., Thijssen, E M.H. J., Nijdam, T.,

Van Rhenen, D. J. $143 \quad$ Van Rhenen, D. J 143

Fettinfusion bei polytraumatisierten Intensivpatienten Effects of Fat Infusion in Patients

after

in der Frühphase der Behandlung Multiple Trauma

Gottardis, M., Hackl, J. M., Gruber, E., Wieser, Chr., Adelsmayr, E., $\quad$ Gottardis, M., Hackl, J. M., Gruber, E., Wieser, Chr., Adelsmayr, E.,

Seyr,M., ZadravecM., Ffeiffer, K.F. $150 \quad$ Seyr, M., Zadravec M., Ffeiffer, K.E 150

Perioperative Glukose-Insulin-Kalium-Infusion in der Perioperative Glucose-Insulin-

Potassium Infusion in

elektiven koronaren Bypass-Chirurgie: Nur geringer Nutzen $\quad$ Elective Coronary Surgery:

Minor Benefit in Connection

bei gleichzeitiger Blutkardioplegie? with Blood Cardioplegia?

Wistbacka, J.-O. M., Nuutinen, L. S., Lepojärvi, M.V. K., Nissinen, J., $\quad$ Wistbacka, J.-O. M., Nuutinen, L. S., Lepojärvi, M. V. K., Nissinen, J.,

Karlqvist, K.E.V., Ruokonen, A 160 Karlqvist, K.E. V., Ruokonen, A 160

Übersichtsarbeit Review Article

Immunmodulation durch mehrfach ungesättigte Immune Modulation by Polyunsaturated

Fatty Acids during 
Fettsäuren in der Ernährungstherapie: Einfluß auf Synthese Nutritional Therapy: Interactions with Synthesis and Effects

und Wirkung der Eikosanoide of Eicosanoids

Suchner, U, Senftleben, U. 167 Suchner, U, Senftleben, U. 167

HLA-Genkomplex - Nachweismethoden und HLA Gene Complex - Methods of

Detection and

klinische Bedeutung Clinical Significance

Editorial Editorial

Mayr, W.R $184 \quad$ Mayr,W.R 184

Der HLA-Genkomplex The HLA Gene Complex

Mayr,W.R $185 \quad$ Mayr,W.R 185

Infusionstherapie Transfusionsmedizin

Band 21, Heft 3, Juni 1994

Inhalt Contents

Serologischer Nachweis von HLA-Antigenen und Serological Detection of HLA Antigens and HLA-Antikörpern HLA Antibodies

Mueller-Eckhardt, G 192 Mueller-Eckhardt, G 192

Einfluß der HLA-Kompatibilität auf die Influence of HLA Compatibility on Kidney

Nierentransplantation Transplantation

Opelz, G., Wujciak, T., Back, D., Mytilineos, J., Schwarz, V., Opelz, G., Wujciak, T., Back, D., Mytilineos, X, Schwarz, V.,

Albrecht, G 198 Albrecht, G 198

Knochenmarktransplantation Bone Marrow Transplantation

Eiermann, T.H203 Eiermann, T.H 203

HLA und Transfusion HLA and Transfusion

Wittmann, G, Zimmermann, R., Eckstein, R 207 Wittmann, G., Zimmermann, R., Eckstein, R 207

HLA und Krankheitsassoziationen HLA and Disease Associations

Wegener, S 213 Wegener, S 213

Gesellschaftsmitteilungen 221 Society Bulletins 221

Lmpressum 133 Imprint 133

Hinweise für Autoren 232 Instructions to Authors 232

Bibliographischer Hinweis: Inhaltsverzeichnisse dieser Zeitschrift erscheinen regelmäßig in current contents ${ }^{\circledR}$ sowie in anderen bibliographischen Diensten. 